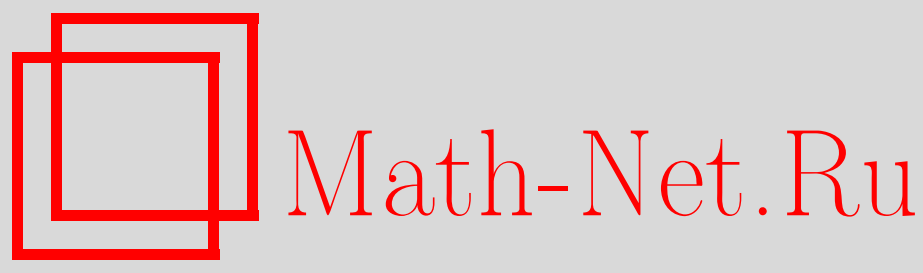

М. А. Браун, Об энергии Казимира в среде с дисперсией и поглощением в рамках подхода диагонализации Фано, TMФ, 2017, том 190, номер 2, 277-292

DOI: https://doi.org/10.4213/tmf9111

Использование Общероссийского математического портала Math-Net.Ru подразумевает, что вы прочитали и согласны с пользовательским соглашением http://www . mathnet.ru/rus/agreement

Параметры загрузки:

IP : 3.93 .64 .190

26 апреля 2023 г., 17:18:08

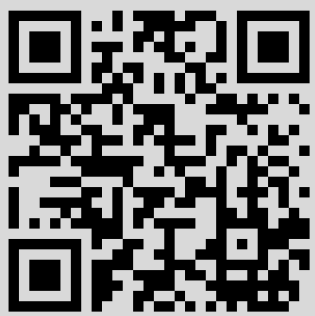




\title{
ФИЗИКА
}

Том 190, № 2

февраль, 2017

(C) 2017 г.

М. А. Браун*

\section{ОБ ЭНЕРГИИ КАЗИМИРА В СРЕДЕ С ДИСПЕРСИЕЙ И ПОГЛОЩЕНИЕМ В РАМКАХ ПОДХОДА ДИАГОНАЛИЗАЦИИ ФАНО}

\begin{abstract}
Энергия Казимира для электромагнитного поля в одномерном пространстве между двумя металлическими пластинами, заполненными диэлектриком с дисперсией и поглощением, вычислена в рамках микроскопического подхода, в котором среда моделируется набором осцилляторов с непрерывно распределенными частотами. Проанализирована трактовка сингулярных выражений, используемых в других работах, и показано, что при подходящем выборе калибровки и отбрасывании некоторых бесконечных членов результаты совпадают с теми, которые получаются при использовании подхода, где такие сингулярности отсутствуют. Изучено асимптотическое поведение на больших расстояниях, и сделан вывод, что оно всегда соответствует притяжению. Однако на конечных расстояниях влияние диэлектрика может приводить к отталкиванию.
\end{abstract}

Ключевые слова: квантовая электродинамика, среда с дисперсией и поглощением, энергия и силы Казимира.

DOI: $10.4213 / \operatorname{tmf} 9111$

\section{1. ВВЕДЕНИЕ}

Последовательный вывод сил Казимира в диэлектрике с дисперсией и поглощением требует включения поглощающей среды в качестве самостоятельной динамической системы. Эта проблема привлекла к себе внимание уже давно. Формализм, позволяющий рассматривать поглощение электромагнитного поля средой в микроскопическом подходе, был развит Хуттнером и Барнеттом [1]. Он заключается в моделировании среды набором осцилляторов с непрерывно распределенными частотами. Взаимодействие со средой ведет к поглощению электромагнитного поля и его исключению как независимой динамической переменной. В результате система полностью описывается набором эффективных осцилляторов, которые также

Работа поддержана Санкт-Петербургским государственным университетом (грант № 11.38.197.2014).

* Санкт-Петербургский государственный университет, Санкт-Петербург, Россия. E-mail: m.braun@spbu.ru 
имеют непрерывно распределенные частоты. Возбуждения квантованного поля выражаются через возбуждения этих эффективных осцилляторов ("поляритонов").

В рамках этой или сходной модели, сформулированой явно или подразумеваемой, можно получить выражение для энергии Казимира, используя макроскопические формулы и интерпретируя в них электромагнитное поле как квантовый оператор, подчиняющийся уравнениям Гейзенберга, в которых влияние среды представлено “квантовым шумом", ответственным за поглощение (см. работы [2]-[5]). Проблемой остается полный учет взаимодействия между полем и средой, а также определение основного состояния, которое может изменяться за счет этого взаимодействия.

Последовательный подход предполагает нахождение основного состояния полного гамильтониана и среднего значения в нем. Эта программа была реализована в различных подходах в работах [4], [6]-[9]. В нашей работе [9] энергия Казимира вычислялась непосредственным усреднением гамильтониана, выраженного через операторы рождения и уничтожения эффективных осцилляторов. В других работах используется представление гамильтониана через операторы поля и их производные по времени. При этом в отличие от нашего подхода возникают сингулярные выражения, которые требуют не только регуляризации, но и отбрасывания некоторых бесконечных членов.

Возникает задача сравнения этих подходов с целью проверки обоснованности трактовки сингулярных выражений и ликвидации бесконечностей. Эта задача и решается в настоящей работе. Для простой модели электромагнитного поля в одномерном пространстве между двумя металлическими пластинами, заполненными диэлектриком с дисперсией и поглощением, мы вычисляем энергию Казимира, используя оба подхода: через операторы рождения и поглощения и через поля и их производные. Во втором случае мы используем некоторую естественную регуляризацию и отбрасываем бесконечности, следуя статье [7]. В результате мы находим, что оба пути приводят к одному и тому же значению для энергии Казимира. При этом во втором случае мы получаем выражение, которое совпадает по форме с результатом статьи [7], явно переписанным для нашего случая однородного диэлектрика в конечном интервале, но отличается от результата для однородной среды, полученного в работе [4], [6].

Кроме того, для иллюстрации мы численно определяем энергию Казимира, имея в виду поведение на малых и больших расстояниях между пластинами и возможность возникновения сил отталкивания на некотором интервале расстояний. Эти вычисления также исправляют численные результаты, полученные в нашей работе [9], в которых оказалось неучтенным одно слагаемое в гамильтониане.

\section{2. МОДЕЛЬ}

Как отмечено во введении, модель квантования электромагнитного поля в среде с дисперсией и поглощением была предложена Хуттнером и Барнеттом уже давно [1]. В ней электромагнитное поле непосредственно взаимодействует с атомными осцилляторами, которые в свою очередь взаимодействуют со средой, реализуя дисперсию и поглощение. Эта модель была упрощена Филбиным, который убрал промежуточные осцилляторы, позволяя электромагнитному полю непосредственно взаимодействовать со средой. Результаты оригинальной модели Хуттнера-Барнетта 
можно легко получить из модели Филбина ее последовательным применением сначала к осцилляторам, а затем к электромагнитному полю. По этой причине мы используем заметно более простую модель Филбина.

С целью дальнейшего упрощения мы расматриваем диэлектрик, однородный по крайней мере в части среды, представленный на микроскопическом уровне полем $\mathbf{Y}_{\omega}$ с непрерывно распределенными частотами. Лагранжева плотность распадается на три части: $\mathcal{L}=\mathcal{L}_{\mathrm{e}}+\mathcal{L}_{\mathrm{r}}+\mathcal{L}_{\mathrm{i}}$. Здесь $\mathcal{L}_{\text {е }}$ есть лагранжева плотность электромагнитного поля:

$$
\mathcal{L}_{\mathrm{e}}=\frac{1}{2}\left(\dot{\mathbf{E}}^{2}-\mathbf{B}^{2}\right), \quad \mathbf{E}=-\dot{\mathbf{A}}-\nabla \phi, \quad \mathbf{B}=\nabla \times \mathbf{A},
$$

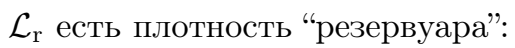

$$
\mathcal{L}_{\mathrm{r}}=\frac{1}{2} \int_{0}^{\infty} d \omega\left(\dot{\mathbf{Y}}_{\omega}^{2}-\omega^{2} \mathbf{X}^{2}\right)
$$

и, наконец, $\mathcal{L}_{\mathrm{i}}$ есть лагранжева плотность взаимодействия, которую мы выбираем, следуя работе [7]:

$$
\mathcal{L}_{\mathrm{i}}=-\int_{0}^{\infty} d \omega v(\omega) \mathbf{A} \dot{\mathbf{Y}}_{\omega}
$$

где $v^{2}(\omega)$ есть квадратично интегрируемая функция, аналитически продолжаемая на отрицательные значения $\omega$ как четная функция. В отличие от Филбина мы не вводим взаимодействия ни со скалярным потенциалом $\phi$, ни с магнитным полем, предполагая среду магнитно нейтральной.

В кулоновской калибровке динамическая часть плотности лагранжиана оказывается выраженной через поперечные поля. В импульсном пространстве лагранжиан представляется суммой по двум поляризациям $\lambda=1,2$ :

$$
L_{\lambda}=\int \frac{d^{3} k}{(2 \pi)^{3}}\left\{\frac{1}{2}\left(\left|\dot{A}_{\lambda}\right|^{2}-k^{2}\left|A_{\lambda}\right|^{2}+\int_{0}^{\infty} d \omega\left|\dot{Y}_{\omega, \lambda}\right|^{2}-\omega^{2}\left|Y_{\omega, \lambda}\right|^{2}\right)-\int_{0}^{\infty} d \omega v(\omega) X_{\lambda}^{*} \dot{Y}_{\omega, \lambda}\right\} .
$$

Здесь мы делаем дополнительное упрощение, переходя к одномерному ограниченному пространству $0<x<a$ и накладывая граничные условия для двух металлических пластин

$$
A(x=0)=A(x=a)=Y_{\omega}(x=0)=Y_{\omega}(x=a)=0 .
$$

Тогда лагранжиан становится суммой по дискретным значениям импульса (модам) $k_{n}=\pi n / a, n=1,2, \ldots$, и поля представляются как

$$
A(x)=\sqrt{\frac{2}{a}} \sum_{n} A_{n} \sin \left(k_{n} x\right), \quad Y_{\omega}(x)=\sqrt{\frac{2}{a}} \sum_{n} Y_{n \omega} \sin \left(k_{n} x\right) .
$$

После этого квантование совершается стандартным образом посредством введения сопряженных полей $\pi(x)$ и $\Pi_{\omega}(x)$ для $A(x)$ и $Y_{\omega}(x)$ соответственно и накладыванием стандартных коммутационных сооношений.

Можно получить два эквивалентных выражения для полного гамильтониана. В терминах полей и их производных по времени

$$
H=\frac{1}{2} \sum_{n}\left\{\dot{A}_{n}^{2}+k_{n}^{2} A_{n}^{2}+\int_{0}^{\infty} d \omega\left(\dot{Y}_{n, \omega}^{2}+\omega^{2} Y_{n, \omega}^{2}\right)\right\}
$$


а в терминах полей и их сопряженных импульсов

$$
H=\frac{1}{2} \sum_{n}\left(\pi_{n}^{2}+k_{1 n}^{2} A_{n}^{2}+\int_{0}^{\infty} d \omega\left(\Pi_{n, \omega}^{2}+\omega^{2} Y_{n, \omega}^{2}\right)+\sum_{n} \int_{0}^{\infty} d \omega v(\omega) A_{n} \Pi_{n, \omega}\right),
$$

где

$$
k_{1 n}^{2}=k_{n}^{2}+\int_{0}^{\infty} d \omega v^{2}(\omega) .
$$

Мы перешли к ограниченному одномерному пространству с целью сравнения со стандартным выводом энергии Казимира. Переход к бесконечному трехмерному пространству требует замены в окончательных формулах, имеющих вид

$$
\sum_{n}(\cdot) \mapsto \int \frac{d^{3} k}{(2 \pi)^{3}}(\cdot)
$$

\section{3. ДИАГОНАЛИЗАЦИЯ ФАНО,}

\section{ЭНЕРГИЯ ОСНОВНОГО СОСТОЯНИЯ И ЭНЕРГИЯ КАЗИМИРА}

В терминах операторов рождения и уничтожения $a_{n}, a_{n}^{\dagger}$ для электромагнитного поля и $b_{n, \omega}, b_{n, \omega}^{\dagger}$ для среды гамильтониан имеет вид

$$
H=\frac{1}{2} \sum_{n}\left[k_{1 n}\left\{a_{n}^{\dagger}, a_{n}\right\}+\int_{0}^{\infty} d \omega \omega\left\{b_{n \omega}^{\dagger}, b_{n \omega}\right\}+\frac{1}{2} \int_{0}^{\infty} d \omega V_{n}(\omega)\left[a_{n}^{\dagger}+a_{n}\right]\left[b_{n \omega}^{\dagger}+b_{n \omega}\right]\right] .
$$

Здесь $V_{n}(\omega)=\sqrt{\omega / k_{1 n}} v(\omega)$. Можно показать, что гамильтониан $H$ можно диагонализовать посредством введения новых полевых переменных [1]

$$
B_{n, \omega}=\alpha_{0 n}(\omega) a_{n}+\beta_{0 n}(\omega) a_{n}^{\dagger}+\int_{0}^{\infty} d \omega^{\prime}\left[\alpha_{1 n}\left(\omega, \omega^{\prime}\right) b_{n, \omega^{\prime}}+\beta_{1 n}\left(\omega, \omega^{\prime}\right) b_{n, \omega^{\prime}}^{\dagger}\right]
$$

которые удовлетворяют коммутационным соотношениям

$$
\left[B_{n, \omega}, B_{n^{\prime}, \omega^{\prime}}^{\dagger}\right]=\delta_{n n^{\prime}} \delta\left(\omega-\omega^{\prime}\right), \quad\left[B_{n, \omega}, B_{n^{\prime}, \omega}\right]=0 .
$$

Вместе с требованием

$$
\left[B_{n, \omega}, H\right]=\omega B_{n, \omega}
$$

они однозначно определяют коэффициенты $\alpha_{0 n}, \beta_{0 n}, \alpha_{1 n}, \beta_{1 n}$ (см. статью [1]):

$$
\begin{gathered}
\alpha_{0 n}(\omega)=-\left(\frac{\omega+k_{1 n}}{2}\right) V_{n}(\omega) P_{n}^{*}(\omega), \quad \beta_{0 n}(\omega)=-\left(\frac{\omega-k_{1 n}}{2}\right) V_{n}(\omega) P_{n}^{*}(\omega), \\
\alpha_{1 n}\left(\omega, \omega^{\prime}\right)=\delta\left(\omega-\omega^{\prime}\right)-\frac{k_{1 n}}{2}\left(\frac{V_{n}^{*}\left(\omega^{\prime}\right) V_{n}(\omega)}{\omega-\omega^{\prime}-i 0}\right) P_{n}^{*}(\omega) \\
\beta_{1}\left(\omega, \omega^{\prime}\right)=-\frac{k_{1 n}}{2}\left(\frac{V_{n}\left(\omega^{\prime}\right) V_{n}(\omega)}{\omega+\omega^{\prime}}\right) P_{n}^{*}(\omega) .
\end{gathered}
$$

Здесь

$$
P_{n}(\omega)=\frac{1}{k^{2}-\epsilon(\omega) \omega^{2}}
$$


есть пропагатор электромагнтного поля в среде, а

$$
\epsilon(\omega)=1+\frac{k_{1 n}}{\omega} \int_{-\infty}^{\infty} \frac{d \omega^{\prime}}{\omega^{\prime}} \frac{\left|V_{n}\left(\omega^{\prime}\right)\right|^{2}}{\omega^{\prime}-\omega-i 0}
$$

есть диэлектрическая постоянная.

В терминах новых переменных гамильтониан $H$ приобретает вид

$$
H=\sum_{n} \int_{0}^{\infty} d \omega \omega B_{n, \omega}^{\dagger} B_{n, \omega}+E(a) .
$$

Здесь $E(a)$ - константа, которая, как очевидно, имеет смысл энергии основного состояния системы.

Предполагая, что новые операторы $B_{n, \omega}$ и $B_{n, \omega}^{\dagger}$ образуют полный набор, можно обратить соотношения (10) и сопряженные к ним и выразить исходные операторы как линейные суперпозиции новых. Сравнивая коммутационные соотношения между старыми и новыми операторами, записанные в терминах старых и новых операторов, можно найти, что

$$
\begin{gathered}
a_{n}=\int_{0}^{\infty} d \omega\left[\alpha_{0 n}^{*}(\omega) B_{n, \omega}-\beta_{0 n}(\omega) B_{n, \omega}^{\dagger}\right], \\
b_{n, \omega}=\int_{0}^{\infty} d \omega^{\prime}\left[\alpha_{1 n}^{*}\left(\omega^{\prime}, \omega\right) B_{n, \omega^{\prime}}-\beta_{1 n}\left(\omega^{\prime}, \omega\right) B_{n, \omega^{\prime}}^{\dagger}\right] .
\end{gathered}
$$

Выражая теперь старые операторы $A_{n}, Y_{n, \omega}$, их сопряженные импульсы и производные по времени, а затем находя среднее в основном состоянии, определяемом условием $B_{n, \omega}|0\rangle=0$, можно найти энергию основного состояния $E_{0}$. Для этого воспользуемся любой из форм (5) или (6). Окончательные формулы выглядят по-разному, но расчеты показывают, что они естественно приводят к одинаковым результатам.

Простейший вывод основан на выражении (6). Энергия основного состояния для данной моды $n$ представляется суммой четырех членов:

$$
E_{n}=\frac{1}{2}\left(k_{1 n}-k_{n}\right)+\left\langle H_{A}\right\rangle_{n}+\left\langle H_{1}\right\rangle_{n}+\left\langle H_{\mathrm{I}}\right\rangle_{n},
$$

где $\left\langle H_{A}\right\rangle_{n},\left\langle H_{Y}\right\rangle_{n}$ и $\left\langle H_{\mathrm{I}}\right\rangle_{n}$ суть энергии основного состояния для электромагнитного поля, поля $Y$ и их взаимодействия соответственно. Явные выражения для них имеют вид

$$
\begin{gathered}
\left\langle H_{A}\right\rangle_{n}=\frac{1}{2 \pi} \int_{0}^{\infty} d \omega\left(\omega-k_{1 n}\right)^{2} \operatorname{Im} P_{n}(\omega) \\
\left\langle H_{\mathrm{I}}\right\rangle_{n}=\frac{1}{\pi} \int_{0}^{\infty} d \omega\left(\omega^{2}-k_{1 n}^{2}\right) \operatorname{Im} P_{n}(\omega) \\
\left\langle H_{Y}\right\rangle_{n}=\frac{1}{2 \pi} \int_{0}^{\infty} d \omega J(\omega) \operatorname{Im} P_{n}(\omega)
\end{gathered}
$$

где

$$
J(\omega)=\int_{0}^{\infty} d \omega^{\prime} \frac{\omega^{\prime 2} \rho\left(\omega^{\prime}\right)}{\left(\omega^{\prime}+\omega\right)^{2}}
$$


и мы обозначили $\rho(\omega)=|v(\omega)|^{2}$. Положительным качеством этих формул является то, что их вывод осуществляется непосредственно и при этом мы не встречаем произведений сингулярных функций. Поэтому результат является вполне однозначным. Однако эти выражения непрозрачны и их отношение к диэлектрической постоянной неочевидно.

Альтернативный вывод, использованный в статье [7] для сред с гладкой неоднородностью, основан на выражении (5). Окончательные формулы в этом случае оказываются непосредственно выраженными через диэлектрическую постоянную. Однако ценой за это является то, что при выводе приходится выделять конечные члены из первоначально сингулярных выражений и прибегать к промежуточной регуляризации, что может приводить к определенной неоднозначности. Для ее устранения, а также для применения к случаю двух параллельных металлических пластин в следующем разделе мы выводим энергию Казимира, основываясь на выражении (5) и используя оригинальную технику. Результаты оказываются теми же, что получились бы из формулы, полученной в статье [7], при ее прямом обобщении на наш случай.

\section{4. ЭНЕРГИЯ ОСНОВНОГО СОСТОЯНИЯ ДЛЯ ГАМИЛЬТОНИАНА В ФОРМЕ (5)}

4.1. Часть $H$, связанная с электромагнитным полем. Часть $H$, происходящая от электромагнитного поля (в расчете на одну моду $n$ ), определяется формулой

$$
H_{A}=\frac{1}{2}\left(\dot{A}_{n}^{2}+k_{n}^{2} A_{n}^{2}\right) .
$$

Мы имеем

$$
A_{n}=\frac{1}{\sqrt{2 k_{1 n}}} \int_{0}^{\infty} d \omega\left[\gamma_{0 n}^{*}(\omega) B_{n, \omega}+\text { э. c. }\right] \text {, }
$$

и в результате

$$
\dot{A}_{n}=-\frac{i}{\sqrt{2 \omega}} \int_{0}^{\infty} d \omega \omega\left[\gamma_{0 n}^{*}(\omega) B_{n, \omega}-\text { э. c. }\right] .
$$

В терминах операторов $B_{n, \omega}$ и $B_{n, \omega}^{\dagger}$ получаем, что $H_{A}=H_{A}^{(1)}+H_{A}^{(2)}$, где

$$
\begin{aligned}
H_{A}^{(1)}=-\frac{1}{2} \frac{1}{2 k_{1 n}} \int_{0}^{\infty} d \omega_{1} \omega_{1} \int_{0}^{\infty} d \omega_{2} \omega_{2}\left[\gamma_{0 n}^{*}\left(\omega_{1}\right) B_{n, \omega_{1}}-\gamma_{1 n}\left(\omega_{1}\right) B_{n, \omega_{1}}^{\dagger}\right] \times \\
\times\left[\gamma_{1 n}^{*}\left(\omega_{2}\right) B_{n, \omega_{2}}-\gamma_{1 n}\left(\omega_{2}, \omega\right) B_{n, \omega_{2}}^{\dagger}\right]
\end{aligned}
$$

и

$$
\begin{aligned}
H_{A}^{(2)}=\frac{1}{2} \frac{k_{n}}{2 k_{1 n}} \int_{0}^{\infty} d \omega_{1} \int_{0}^{\infty} d \omega_{2}\left[\gamma_{0 n}^{*}\left(\omega_{1}\right) B_{n, \omega_{1}}\right. & \left.+\gamma_{0 n}\left(\omega_{1}\right) B_{n, \omega_{1}}^{\dagger}\right] \times \\
& \times\left[\gamma_{0 n}^{*}\left(\omega_{2}\right) B_{n, \omega_{2}}+\gamma_{0 n}\left(\omega_{2}\right) B_{n, \omega_{2}}^{\dagger}\right] .
\end{aligned}
$$

Вычисляя среднее в основном состоянии, получаем равенство

$$
\left\langle H_{A}\right\rangle_{n}=\frac{1}{2} \int_{0}^{\infty} d \omega \frac{\omega^{2}+k_{n}^{2}}{2 k_{1 n}}\left|\gamma_{0 n}(\omega)\right|^{2} .
$$


Из наших формул следует выражение

$$
\left|\gamma_{0 n}(\omega)\right|^{2}=k_{1 n}^{2} V_{n}^{2}(\omega)\left|P_{n}(\omega)\right|^{2}=\frac{2}{\pi} k_{1 n} \operatorname{Im} P_{n}\left(\omega_{1}\right),
$$

что приводит к окончательному результату

$$
\left\langle H_{A}\right\rangle_{n}=\frac{1}{2 \pi} \int_{0}^{\infty} d \omega\left(\omega^{2}+k_{n}^{2}\right) \operatorname{Im} P_{n}(\omega) .
$$

4.2. Часть $H$, связанная со средой. Часть $H$, происходящая за счет среды, в расчете на одну моду есть

$$
H_{Y}=\frac{1}{2} \int_{0}^{\infty} d \omega\left(\dot{Y}_{n, \omega}^{2}+\omega^{2} Y_{n, \omega}^{2}\right)
$$

Как и для электромагнитного поля, находим, что

$$
\left\langle H_{Y}\right\rangle_{n}=\frac{1}{2} \sum_{n} \int_{0}^{\infty} d \omega \frac{1}{2 \omega} \int_{0}^{\infty} d \omega_{1}\left(\omega^{2}+\omega_{1}^{2}\right)\left|\gamma_{1 n}\left(\omega_{1}, \omega\right)\right|^{2} .
$$

K сожалению, $\gamma_{1 n}\left(\omega_{1}, \omega\right)$ сингулярна:

$$
\gamma_{1 n}\left(\omega_{1}, \omega\right)=\delta\left(\omega_{1}-\omega\right)-a_{n}\left(\omega_{1}, \omega\right)\left(\frac{1}{\omega_{1}-\omega-i 0}+\frac{1}{\omega_{1}+\omega}\right)
$$

где

$$
a_{n}\left(\omega_{1}, \omega\right)=\frac{1}{2} k_{1 n} V_{n}\left(\omega_{1}\right) P_{n}^{*}\left(\omega_{1}\right) V_{n}(\omega)
$$

Поэтому $\left|\gamma_{1 n}\left(\omega_{1}, \omega\right)\right|^{2}$ не существует из-за членов, сингулярных при $\omega=\omega_{1}$. Однако оказывается, что если отбросить некоторые бесконечные члены, то оставшиеся сингулярные члены сокращаются.

Следуя статье [7], мы отбрасываем член с квадратом $\delta\left(\omega_{1}-\omega\right)$. Оставшиеся члены мы разобьем на две части:

$$
\begin{aligned}
T_{1}=-\frac{1}{2} \int_{0}^{\infty} d \omega \frac{1}{2 \omega} \int_{0}^{\infty} & d \omega_{1}\left(\omega^{2}+\omega_{1}^{2}\right) \delta\left(\omega_{1}-\omega\right) \times \\
\times & {\left[a_{n}\left(\omega_{1}, \omega\right)\left(\frac{1}{\omega_{1}-\omega-i 0}+\frac{1}{\omega_{1}+\omega}\right)+\text { к. с. }\right] }
\end{aligned}
$$

и

$$
T_{2}=\frac{1}{2} \int_{0}^{\infty} d \omega \frac{1}{2 \omega} \int_{0}^{\infty} d \omega_{1}\left(\omega^{2}+\omega_{1}^{2}\right)\left|a_{n}\left(\omega_{1}, \omega\right)\right|^{2}\left|\frac{1}{\omega_{1}-\omega-i 0}+\frac{1}{\omega_{1}+\omega}\right|^{2} .
$$

Для анализа сокращений сингулярных членов мы вынуждены регуляризовать наши фунции. Для этого выбираем

$$
\begin{gathered}
\frac{1}{\omega_{1}-\omega-i 0} \mapsto \frac{1}{\omega_{1}-\omega-i \epsilon}, \\
\delta\left(\omega_{1}-\omega\right)=\frac{1}{2 \pi i}\left(\frac{1}{\omega_{1}-\omega-i \epsilon}-\frac{1}{\omega_{1}-\omega+i \epsilon}\right)
\end{gathered}
$$

с малым конечным положительным $\epsilon$. 
В приложении мы показываем, что сингулярные члены, пропорциональные $1 / \epsilon$, сокращаются в сумме $T_{1}$ и $T_{2}$. Там же вычислены и конечные вклады $F_{1}$ и $F_{2}$, происходящие от этих двух частей. Вычисляя их сумму, получаем, что

$$
\begin{aligned}
\left\langle H_{Y}\right\rangle_{n} & =\frac{1}{2 \pi} \int_{0}^{\infty} d \omega \omega^{2}\left\{[\omega \operatorname{Im}(\epsilon(\omega)-1)]^{\prime} \operatorname{Re} P_{n}(\omega)+\left([\omega \operatorname{Re}(\epsilon(\omega)-1)]^{\prime}\right) \operatorname{Im} P_{n}(\omega)\right\}= \\
& =\frac{1}{2 \pi} \int_{0}^{\infty} d \omega \omega^{2} \operatorname{Im}\left([\omega(\epsilon(\omega)-1)]^{\prime} P_{n}(\omega)\right) .
\end{aligned}
$$

Сумма $\left\langle H_{A}\right\rangle_{n}$ и $\left\langle H_{y}\right\rangle_{n}$ дает полную энергию основного состояния в расчете на одну моду:

$$
E_{0 n}=\frac{1}{2 \pi} \int_{0}^{\infty} d \omega \operatorname{Im}\left(\omega^{2} \frac{d}{d \omega}[\omega \epsilon(\omega)]+k_{n}^{2}\right) P_{n}(\omega) .
$$

Это выражение совпадает с формулой в [7], подходящим образом преобразованной к магнитно-нейтральному диэлектрику между двумя параллельными металлическими пластинами.

Численные расчеты показывают, что энергия Казимира, получающаяся из (35), в точности совпадает с той, что получается из (20).

\section{5. ЭНЕРГИЯ КАЗИМИРА. АСИМПТОТИКИ И ЧИСЛЕННЫЕ РАСЧЕТЫ}

Энергию Казимира можно получить как разность между энергией в интервале $0 \leqslant x \leqslant a$ и ее значением в вакууме в том же интервале. Она определяется разностью между суммой по всем модам $n$ и интегралом по значениям $k$, который дает энергию в интервале $0 \leqslant x \leqslant a$ в вакууме:

$$
E_{\text {Cas }}=\sum_{n=1}^{\infty} E_{0 n}-\int_{0}^{\infty} d n E_{0 k}
$$

где $E_{0 k}$ получается из (35) подстановкой $k_{n} \rightarrow \pi n / a$.

$\mathrm{C}$ точностью до бесконечного слагаемого, не зависящего от $a$ и поэтому не влияющего на силы Казимира, выражение (35) можно переписать в виде

$$
E_{0 n}=\frac{1}{2 \pi} \int_{0}^{\infty} d \omega \operatorname{Im} \omega^{2}\left(\frac{d}{d \omega}[\omega \epsilon(\omega)]+\epsilon(\omega)\right) P_{n}(\omega) .
$$

Чтобы обеспечить возможность разворота контура на мнимую ось, мы вычисляем разность $\Delta E_{\text {Cas }}$ между энергией Казимира в среде и в вакууме. Суммирование по модам можно выполнить с помощью формулы [10]

$$
\gamma \sum_{n=1}^{\infty} \frac{1}{n^{2}+\gamma}=\frac{\pi}{2} \operatorname{cth}(\pi \gamma)-\frac{1}{2 \gamma}
$$

Это дает энергию Казимира, связанную с наличием диэлектрика, в виде

$$
\Delta E_{\mathrm{Cas}}=-\frac{1}{2 \pi} \int_{0}^{\infty} d \xi \xi^{2}(\rho(\xi) W(q)-2 W(\xi)) .
$$


Здесь

$$
\rho(\xi)=\left(\frac{d}{d \omega}[\omega \epsilon(\omega)]+\epsilon(\omega)\right)_{\omega=i \xi}
$$

и

$$
W(q)=\frac{a}{2 q}\left(\operatorname{cth}(a q)-1-\frac{1}{a q}\right), \quad q^{2}=\xi^{2} \epsilon(i \xi) .
$$

Полная энергия Казимира есть сумма (39) и вакуумного вклада $E_{\mathrm{Cas}}^{(0)}=-\pi / 24 a$.

Переходя к интегрированию по $q$, преобразуем (39) в формулу

$$
\Delta E_{\mathrm{Cas}}=\frac{1}{\pi} \int_{0}^{\infty} d q q W(q, a)(q-\xi(q)),
$$

где $\xi$ следует выразить через $q$. Используя формулу $(41)$, разлагаем $\Delta E_{\text {Cas }}$ в сумму $\Delta E_{\mathrm{Cas}}=\Delta E_{\mathrm{Cas}}^{(1)}+\Delta E_{\mathrm{Cas}}^{(2)}$, где

$$
\Delta E_{\mathrm{Cas}}^{(1)}=\frac{a}{2 \pi} \int_{0}^{\infty} d q(q-\xi(q)(\operatorname{cth}(a q)-1)),
$$

a

$$
\Delta E_{\mathrm{Cas}}^{(2)}=\frac{1}{2 \pi} \int_{0}^{\infty} \frac{d q}{q}(q-\xi(q)(\operatorname{cth}(a q)-1))
$$

и не зависит от $a$.

Легко найти, что $\Delta E_{\text {Cas }}=0$ в точке $a=0$. Поскольку $\operatorname{cth}(a q)-1$ положительно и убывает с ростом $a q$, мы находим, что $\Delta E_{\text {Cas }}$ отрицательна и убывает с ростом $a$, что означает наличие силы отталкивания.

Асимптотическое поведение при больших $а$ зависит от поведения $\epsilon(\omega)$ при $\omega \rightarrow 0$. Если $\operatorname{Im} \epsilon(\omega) \sim 1 / \omega$ (случай A), тогда при малых $\xi$ находим, что $\beta(\xi)=c_{\mathrm{A}} \xi$. Если же $\operatorname{Im} \epsilon(\omega) \sim \omega$ (случай Б), то тогда $\beta(\xi)=c_{\text {Б }} \xi^{2}$. В обоих случаях $c_{\mathrm{A}, \mathrm{b}}>0$. Используя это, легко получить, что в случае А при больших $a$

$$
\Delta E_{\mathrm{Cas}}^{(1)}=\frac{\pi^{2}}{24 a}-\frac{\zeta(3)}{4 \pi c_{\mathrm{A}}} \frac{1}{a^{2}}
$$

Первое слагаемое сокращает вклад вакуума, и полная энергия Казимира (с точностью до константы) ведет себя как $-1 / a^{2}$ (случай притяжения, быстро убывающего с расстоянием).

В случае Б асимптотика $\Delta E_{\text {Саs }}^{(1)}$ такая же, как и у вакуумного вклада (т. е. $\left.\propto-1 / a\right)$ с меньшим коэффициентом и противоположным знаком, так что полная энергия Казимира имеет асимптотику

$$
E_{\mathrm{Cas}}(a)=-\frac{\pi^{2}}{24\left(1+c_{\mathrm{E}}\right) a}, \quad a \rightarrow \infty,
$$

(случай ослабленного притяжения). 


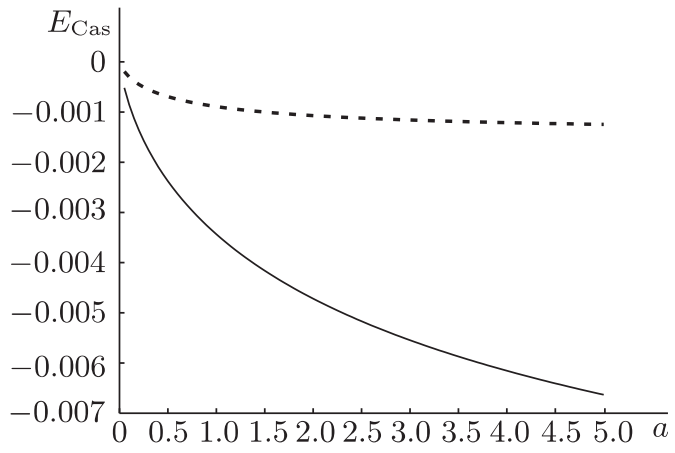

a

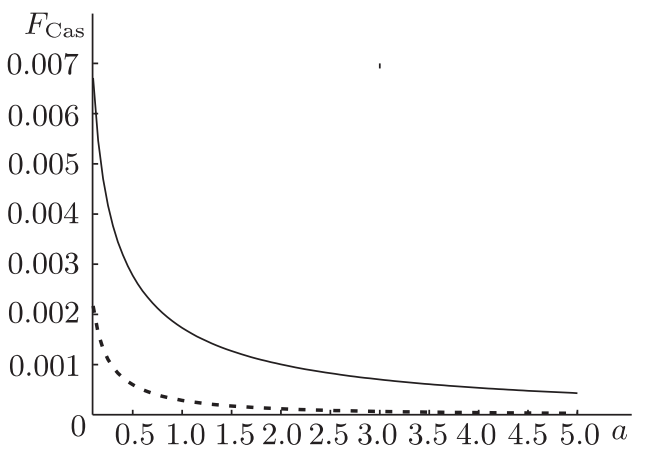

б

Рис. 1. Энергия Казимира (а) и сила (б), связанные с наличием диэлектрика, как функции расстояния $а$ для случаев А (нижние кривые) и Б (верхние кривые).

При малых $а$ энергия Казимира, связанная с диэлектриком, имеет логарифмическое поведение:

$$
\Delta E_{\mathrm{Cas}}=\frac{\beta_{\infty}}{4 \pi} a \ln a,
$$

где $\beta_{\infty}$ - конечное положительное предельное значение $\beta(\xi)$ при $\xi \rightarrow \infty$. В зависимости от силы взаимодействия этот вклад может оказаться доминирующим и привести к силам отталкивания в ограниченной области $a$.

Для илюстрации приложения этой техники мы вычисляем энергию Казимира, связанную с наличием диэлектрика, для двух типичных форм функции связи $v(\omega)$ и следующей из нее $\epsilon(\omega)$, соответствующих случаям А и Б, описанным выше. В случае $\mathrm{A}$

$$
v(\omega)=\frac{g^{2} m^{3}}{\omega^{2}+m^{2}}, \quad \epsilon(i \xi)=\frac{\pi g^{2} m^{2}}{2 \xi(\xi+m)} .
$$

В случае Б

$$
v(\omega)=\frac{g^{2} m^{3} \omega^{2}}{\left(\omega^{2}+m^{2}\right)^{2}}, \quad \epsilon(i \xi)=1+\frac{\pi g^{2} m^{2}}{4(\xi+m)^{2}} .
$$

Здесь параметрами являются $g$ (безразмерный параметр) и $m$ (имеющий размерность массы), которые определяют силу и $\omega$-зависимость взаимодействия с диэлектриком. При наличии единственного масштаба $m$ энергия дается как $E=m f(m a)$. Мы положили $m=1$, так что энергия измеряется в единицах $m$, а расстояния в единицах $1 / m$. Для простоты мы выбрали $g^{2} \pi=1$.

Результаты наших вычислений энергии Казимира, связанной с наличием диэлектрика, показаны на рис. 1а для этих двух случаев. Соответствующая сила (притяжения) показана на рис. $1 б$.

Отметим, что эти результаты отличаются от опубликованных ранее в сходной задаче в статье [9], где опущено первое (тривиальное) слагаемое в (20), которое ошибочно было сочтено несущественным. 


\section{6. ОБСУЖДЕНИЕ}

Для случая диэлектрика между двумя металлическими пластинами в пространстве одного измерения в формулах, полученных в работах [4] и [7], можно провести прямые замены

$$
\int \frac{d^{3} k}{(2 \pi)^{3}}(\cdot) \mapsto \sum_{n}(\cdot), \quad k \mapsto \frac{n \pi}{a} .
$$

Тогда формула, полученная в статье [7], преобразуется в формулу (35), которую мы вывели непосредственно из гамильтониана. Таким образом, прямая подстановка (50) в выражения для плотности энергии, полученные в статьях [4], [6] или [8], дает правильное значение энергии Казимира.

Отметим, что в своих работах [4], [6] Роза, Далвит и Милони вывели выражение для плотности энергии специально для однородной среды в виде

$$
\bar{u}_{\mathrm{RAM}}=\frac{1}{2 \pi^{2}} \int_{0}^{\infty} d \omega \omega^{3} n_{\mathrm{R}}^{2} \frac{d}{d \omega}\left[\omega n_{\mathrm{R}}(\omega)\right]
$$

где $n(\omega)$ есть индекс преломления, $\epsilon(\omega)=n^{2}(\omega)$. В эту формулу входит только действительная часть $n(\omega)$, что дало авторам основание утверждать, что энергия дается той же самой формулой, что и для среды без поглощения. Наши результаты противоречат этому выводу.

\section{Вычисление $\left\langle H_{Y}\right\rangle$, формулы (31) и (32)}

ПРИЛОЖЕНИЕ

Сингулярные члены $T_{1}$. Перепишем $T_{1}$ как

$$
T_{1}=-\frac{1}{2} \sum_{n} \int_{0}^{\infty} d \omega_{1} t_{1 n}\left(\omega_{1}\right)+\text { к. c. }
$$

где

$$
t_{1 n}\left(\omega_{1}\right)=\int_{0}^{\infty} d \omega f_{n}\left(\omega_{1}, \omega\right)\left(\delta\left(\omega_{1}-\omega\right)+\delta\left(\omega_{1}+\omega\right)\right)\left(\frac{1}{\omega_{1}-\omega-i 0}+\frac{1}{\omega_{1}+\omega}\right),
$$

и $f_{n}\left(\omega_{1}, \omega\right)=\left(\omega^{2}+\omega_{1}^{2}\right) a_{n}\left(\omega_{1}, \omega\right) / 2 \omega$.

Для выделения сингулярной части запишем представление

$$
f_{n}\left(\omega_{1}, \omega\right)=f_{n}\left(\omega_{1}, \omega_{1}\right)+\left(\omega_{1}-\omega\right) f_{1 n}\left(\omega_{1}, \omega\right)
$$

и соответственно разбиение $t_{1 n}=t_{1 n}^{(1)}+t_{1 n}^{(2)}$. Сигулярные члены содержатся в $t_{1 n}^{(1)}$. Благодаря симметрии относительно замены $\omega \rightarrow-\omega$ можно записать равенство

$$
t_{1 n}^{(1)}=f_{n}\left(\omega_{1}, \omega_{1}\right) \int_{-\infty}^{\infty} d \omega \delta\left(\omega_{1}-\omega\right)\left(\frac{1}{\omega_{1}-\omega-i 0}+\frac{1}{\omega_{1}+\omega}\right) .
$$

Внутренний интеграл с регуляризованными функциями есть

$$
J=\frac{1}{2 \pi i} \int_{-\infty}^{\infty} d \omega\left(\frac{1}{\omega_{1}-\omega-i \epsilon}-\frac{1}{\omega_{1}-\omega+i \epsilon}\right)\left(\frac{1}{\omega_{1}-\omega-i \epsilon}+\frac{1}{\omega_{1}+\omega-i \epsilon}\right)=\frac{i}{2 \epsilon}+\frac{1}{2 \omega_{1}} .
$$


Сингулярная часть $T_{1}$ оказывается равной

$$
S_{1}=-\frac{i}{4 \epsilon} \int_{0}^{\infty} d \omega_{1} \omega_{1}\left(f_{n}\left(\omega_{1}, \omega_{1}\right)-f_{n}^{*}\left(\omega_{1}, \omega_{1}\right)\right)=\frac{1}{2 \epsilon} \int_{0}^{\infty} d \omega_{1} \omega_{1} \operatorname{Im} a_{n}\left(\omega_{1}, \omega_{1}\right) .
$$

Используя формулу (30), окончательно получаем сингулярную часть $T_{1}$ в виде

$$
S_{1}=-\frac{\pi}{8 \epsilon} k_{1 n}^{2} \int_{0}^{\infty} d \omega_{1} \omega_{1} V_{n}^{4}\left(\omega_{1}\right)\left|P_{n}\left(\omega_{1}\right)\right|^{2} .
$$

Также от слагаемого $t_{1 n}^{(1)}$ возникает конечный вклад

$$
F_{1}^{(1)}=-\frac{1}{4} \int_{0}^{\infty} \frac{d \omega_{1}}{\omega_{1}} f_{n}\left(\omega_{1}, \omega_{1}\right)
$$

Сингулярные члены в $T_{2}$. Перепишем $T_{2}$ в виде

$$
T_{2}=\frac{1}{2} \int_{0}^{\infty} d \omega_{1} \int_{0}^{\infty} d \omega g_{n}\left(\omega_{1}, \omega\right)\left|\frac{1}{\omega_{1}-\omega-i 0}+\frac{1}{\omega_{1}+\omega}\right|^{2},
$$

где

$$
g_{n}\left(\omega_{1}, \omega\right)=\frac{\omega_{1}^{2}+\omega^{2}}{2 \omega}\left|a_{n}\left(\omega_{1} \omega\right)\right|^{2}=\tilde{g}_{n}\left(\omega_{1}\right) \frac{\omega_{1}^{2}+\omega^{2}}{2 \omega} V_{n}^{2}(\omega)
$$

и

$$
\tilde{g}_{n}\left(\omega_{1}\right)=\frac{1}{4} k_{1 n}^{2} V_{n}^{2}\left(\omega_{1}\right)\left|P_{n}\left(\omega_{1}\right)\right|^{2}
$$

Из четырех членов в квадрате модуля сингулярная часть порождается только произведением

$$
\frac{1}{\omega_{1}-\omega-i 0} \frac{1}{\omega_{1}-\omega+i 0} .
$$

Соответственно, только в этом члене запишем представление

$$
g_{n}\left(\omega_{1}, \omega\right)=g_{n}\left(\omega_{1} \omega_{1}\right)+\left(\omega_{1}-\omega\right) g_{1 n}\left(\omega_{1}, \omega\right)
$$

Сингулярный вклад содержится в

$$
T_{2}^{(1)}=\frac{1}{2} \int_{0}^{\infty} d \omega_{1} g_{n}\left(\omega_{1} \omega_{1}\right) \int_{0}^{\infty} d \omega \frac{1}{\omega_{1}-\omega-i 0} \frac{1}{\omega_{1}-\omega+i 0} .
$$

Регуляризованный внутренний интеграл есть

$$
J=\int_{0}^{\infty} d \omega \frac{1}{\omega_{1}-\omega-i \epsilon} \frac{1}{\omega_{1}-\omega+i \epsilon} .
$$

Его можно вычислить поворотом контура на отрицательную действительную полуось и взятием вычета в полюсе при $\omega=\omega_{1}+i \epsilon$ :

$$
J=\frac{\pi}{\epsilon}-\int_{-\infty}^{0} \frac{d \omega}{\left(\omega-\omega_{1}\right)^{2}}=\frac{\pi}{\epsilon}-\frac{1}{\omega_{1}} .
$$

Получаем, что

$$
T_{2}^{(1)}=\frac{1}{2} \int_{0}^{\infty} d \omega_{1} g_{n}\left(\omega_{1} \omega_{1}\right)\left(\frac{\pi}{\epsilon}-\frac{1}{\omega_{1}}\right)
$$


Таким образом, мы находим сингулярный вклад

$$
S_{2}=\frac{\pi}{2 \epsilon} \int_{0}^{\infty} d \omega_{1} \omega_{1}\left|a_{n}\left(\omega_{1}, \omega\right)\right|^{2}=\frac{\pi}{8 \epsilon} k_{1 n}^{2} \int_{0}^{\infty} d \omega_{1} \omega_{1} V_{n}^{4}\left(\omega_{1}\right)\left|P_{n}\left(\omega_{1}\right)\right|^{2} .
$$

Как видно, $S_{1}+S_{2}=0$, так что сингулярные члены в $\left\langle H_{Y}\right\rangle$ и в самом деле сокращаются.

Кроме этого сингулярного члена в $T_{2}^{(1)}$ также содержится и конечный вклад

$$
F_{2}^{(1)}=-\frac{1}{2} \int_{0}^{\infty} d \omega_{1} \frac{g_{n}\left(\omega_{1} \omega_{1}\right)}{\omega_{1}} .
$$

Конечные члены в $T_{1}$. Как мы видели, один конечный член в $T_{1}^{(1)}$ происходит от $t_{1 n}^{(1)}$. Остальные связаны со слагаемым $t_{1 n}^{(2)}$, происходящим от второго члена в (П.3):

$$
t_{1 n}^{(2)}=f_{1 n}\left(\omega_{1}, \omega_{1}\right)=-\left.\frac{\partial f_{n}\left(\omega_{1}, \omega\right)}{\partial \omega}\right|_{\omega=\omega_{1}} .
$$

Таким образом, дополнительный конечный вклад есть

$$
F_{1}^{(2)}=\left.\frac{1}{2} \int_{0}^{\infty} d \omega_{1} \frac{\partial f_{n}\left(\omega_{1}, \omega\right)}{\partial \omega}\right|_{\omega=\omega_{1}}+\text { к. с. }
$$

Полный конечный вклад от $T_{1}$ имеет вид

$$
F_{1}=F_{1}^{(1)}+F_{1}^{(2)}=\frac{1}{2} \int_{0}^{\infty} d \omega_{1}\left(\left.\frac{\partial f_{n}\left(\omega_{1}, \omega\right)}{\partial \omega}\right|_{\omega=\omega_{1}}-\frac{f_{n}\left(\omega_{1}, \omega_{1}\right)}{2 \omega_{1}}\right)+\text { к. c. }
$$

Получаем, что

$$
\frac{\partial}{\partial \omega} f_{n}\left(\omega_{1}, \omega_{1}\right)=\frac{1}{4} k_{1 n} \omega_{1} P^{*}\left(\omega_{1}\right)\left[V_{n}^{2}\right]^{\prime}\left(\omega_{1}\right) .
$$

Используя равенство

$$
V_{n}^{2}(\omega)=\frac{2}{\pi} \frac{\omega_{1}^{2}}{k_{1 n}} \epsilon_{\mathrm{I}}(\omega),
$$

где $\epsilon_{\mathrm{I}}$ есть мнимая часть диэлектрической постоянной, находим, что

$$
\frac{\partial}{\partial \omega} f_{n}\left(\omega_{1}, \omega_{1}\right)=\frac{1}{2 \pi} \omega_{1}^{2} P_{n}^{*}\left(\omega_{1}\right)\left(2 \epsilon_{\mathrm{I}}\left(\omega_{1}\right)+\omega_{1} \epsilon^{\prime}\left(\omega_{1}\right)\right) .
$$

Также справедливо равенство

$$
\frac{f_{n}\left(\omega_{1}, \omega_{1}\right)}{2 \omega_{1}}=\frac{1}{2 \pi} \omega_{1}^{2} P_{n}^{*}\left(\omega_{1}\right) \epsilon_{\mathrm{I}}\left(\omega_{1}\right) .
$$

Это приводит к окончательному выражению

$$
F_{1}=\frac{1}{2 \pi} \int_{0}^{\infty} d \omega \omega^{2}\left[\omega \epsilon_{\mathrm{I}}(\omega)\right]^{\prime} \operatorname{Re} P_{n}(\omega)
$$


Конечные члены в $T_{2}$. Мы используем ту же технику, что и в предыдущем пункте. Квадрат модуля в (П.7) содержит четыре члена

$$
\frac{1}{\omega_{1}-\omega-i 0} \frac{1}{\omega_{1}-\omega+i 0}+\frac{1}{\omega_{1}+\omega}\left(\frac{1}{\omega_{1}-\omega-i 0}+\frac{1}{\omega_{1}-\omega+i 0}\right)+\left(\frac{1}{\omega_{1}+\omega}\right)^{2}
$$

Из них только первый дает вклад в сингулярную часть. Вычисляя $T_{2}^{(1)}$, содержащий эту сингулярную часть, мы представили $g_{n}\left(\omega_{1}, \omega\right)$ в первом слагаемом в виде (П.10), оставив нетронутыми остальные три слагаемые. Вклад от $g_{n}\left(\omega_{1}, \omega_{1}\right)$ в первом слагаемом есть сумма сингулярной части $S_{2}$ и конечной части $F_{2}^{(1)}$. Кроме этого остается еще конечная часть $F_{2}^{(2)}$, имеющая вид

$$
F_{2}^{(2)}=\frac{1}{2} \int_{0}^{\infty} d \omega_{1} \int_{0}^{\infty} d \omega\left[\left(g_{1 n}\left(\omega_{1}, \omega\right)+2 \frac{g_{n}\left(\omega_{1}, \omega\right)}{\omega_{1}+\omega}\right) \mathrm{P} \frac{1}{\omega_{1}-\omega}+\frac{g_{n}\left(\omega_{1}, \omega\right)}{\left(\omega_{1}+\omega\right)^{2}}\right] .
$$

Рассмотрим первый член в подынтегральном выражении в (П.22) с $g_{1 n}$. Вводя регуляризацию, перепишем его как

$$
\frac{1}{2}\left(g_{n}\left(\omega_{1}, \omega\right)-g_{n}\left(\omega_{1}, \omega_{1}\right)\right)\left(\frac{1}{\left(\omega_{1}-\omega-i \epsilon\right)^{2}}+\frac{1}{\left(\omega_{1}-\omega+i \epsilon\right)^{2}}\right) .
$$

Тогда мы получаем $F_{2}^{(2)}$ в виде

$$
F_{2}^{(2)}=\frac{1}{2} \int_{0}^{\infty} d \omega_{1} \tilde{g}_{n}\left(\omega_{1}\right) J\left(\omega_{1}\right)
$$

где $\tilde{g}_{n}$ дается формулой (П.9), а $J\left(\omega_{1}\right)$ есть сумма трех слагаемых:

$$
J=J^{(1)}+J^{(2)}+J^{(3)} .
$$

В $J^{(1)}$ и $J^{(2)}$ входят первый член из первого множителя в (П.23) и вся оставшаяся часть подынтегрального выражения в (П.22), происходящая из частей $g_{n}\left(\omega_{1}, \omega\right)$, содержащих множители $\omega_{1}^{2} / 2 \omega$ и $\omega / 2$ соответственно:

$$
\begin{aligned}
& J^{(1)}\left(\omega_{1}\right)=\frac{1}{2} \omega_{1}^{2} \int_{0}^{\infty} d \omega \frac{V_{n}^{2}(\omega)}{\omega}\left(\frac{1}{\left(\omega_{1}-\omega\right)^{2}}+\frac{1}{\left(\omega_{1}+\omega\right)^{2}}+\frac{2}{\left(\omega_{1}+\omega\right)\left(\omega_{1}-\omega\right)}\right), \\
& J^{(2)}\left(\omega_{1}\right)=\frac{1}{2} \int_{0}^{\infty} d \omega \omega V_{n}^{2}(\omega)\left(\frac{1}{\left(\omega_{1}-\omega\right)^{2}}+\frac{1}{\left(\omega_{1}+\omega\right)^{2}}+\frac{2}{\left(\omega_{1}+\omega\right)\left(\omega_{1}-\omega\right)}\right),
\end{aligned}
$$

и мы выделили второе слагаемое в первом множителе в (П.23) с тривиальной зависимостью от $\omega$ как $J^{(3)}$ :

$$
J^{(3)}\left(\omega_{1}\right)=-\frac{1}{2} \omega_{1} V_{n}^{2}\left(\omega_{1}\right) \int_{0}^{\infty} d \omega\left(\frac{1}{\left(\omega_{1}-\omega-i \epsilon\right)^{2}}+\frac{1}{\left(\omega_{1}-\omega+i \epsilon\right)^{2}}\right)=V_{n}^{2}\left(\omega_{1}\right) .
$$

Этот интеграл легко вычисляется поворотом контура на отрицательную полуось в верхней или нижней полуплоскости. Вклад от $J^{(3)}$ сокращает ранее вычисленную конечную часть $F_{2}^{(1)}$. Поэтому полная конечная часть $T_{2}$ происходит от интегралов $J^{(1)}$ и $J^{(2)}$. В них все особенности при $\omega=\omega_{1}$ должны пониматься как главные значения с

$$
\mathrm{P} \frac{1}{\left(\omega_{1}-\omega\right)^{2}} \equiv \frac{1}{2}\left(\frac{1}{\left(\omega_{1}-\omega-i 0\right)^{2}}+\frac{1}{\left(\omega_{1}-\omega+i 0\right)^{2}}\right) .
$$


Первые два слагаемые в $J^{(1)}$ приводят к формуле

$$
\begin{aligned}
J_{1}^{(1)} & =-\frac{1}{2} \omega_{1}^{2} \frac{\partial}{\partial \omega_{1}} \int_{0}^{\infty} d \omega \frac{V_{n}^{2}(\omega)}{\omega}\left(\frac{1}{\omega_{1}-\omega}+\frac{1}{\omega_{1}+\omega}\right)= \\
& =-\frac{1}{2} \omega_{1}^{2} \frac{\partial}{\partial \omega_{1}} \int_{-\infty}^{\infty} d \omega \frac{V_{n}^{2}(\omega)}{\omega} \frac{1}{\omega_{1}-\omega} .
\end{aligned}
$$

Последнее слагаемое в $J^{(1)}$ можно переписать в виде

$$
J_{2}^{(1)}\left(\omega_{1}\right)=\omega_{1}^{2} \frac{1}{2 \omega_{1}} \int_{-\infty}^{\infty} d \omega \frac{V_{n}^{2}(\omega)}{\omega} \frac{1}{\omega_{1}-\omega} .
$$

В сумме получаем

$$
J^{(1)}=\frac{1}{2} \omega_{1}^{2}\left(\frac{1}{\omega_{1}}-\frac{\partial}{\partial \omega_{1}}\right) \int_{-\infty}^{\infty} d \omega \frac{V_{n}^{2}(\omega)}{\omega} \frac{1}{\omega_{1}-\omega} .
$$

Таким же путем получаем, что

$$
J^{(2)}=\frac{1}{2}\left(\frac{1}{\omega_{1}}-\frac{\partial}{\partial \omega_{1}}\right) \int_{-\infty}^{\infty} d \omega \omega V_{n}^{2}(\omega) \frac{1}{\omega_{1}-\omega} .
$$

Теперь свяжем интегралы по $\omega$ с диэлектрической постоянной. Определим

$$
z\left(\omega_{1}\right)=\int_{-\infty}^{+\infty} d \omega \frac{V_{n}^{2}(\omega)}{\omega} \frac{1}{\omega_{1}-\omega+i 0}=2 \frac{\omega_{1}}{k_{1 n}}\left(1-\epsilon^{*}\left(\omega_{1}\right)\right) .
$$

В смысле главного значения имеем

$$
\operatorname{Re} z\left(\omega_{1}\right)=2 \frac{\omega_{1}}{k_{1 n}}\left(1-\epsilon_{\mathrm{R}}\left(\omega_{1}\right)\right)
$$

где $\epsilon_{\mathrm{R}}$ есть действительная часть диэлектрической постоянной.

Возьмем второй интеграл:

$$
\int_{-\infty}^{+\infty} d \omega \omega V_{n}^{2}(\omega) \frac{1}{\omega_{1}-\omega+i 0}=\int_{-\infty}^{+\infty} d \omega V_{n}^{2}(\omega) \frac{\omega-\omega_{1}+\omega_{1}}{\omega_{1}-\omega+i 0}=\omega_{1} u\left(\omega_{1}\right)
$$

где

$$
u\left(\omega_{1}\right)=\int_{-\infty}^{+\infty} d \omega \frac{V_{n}^{2}(\omega)}{\omega_{1}-\omega+i 0}=2 \frac{\omega_{1}^{2}}{k_{1 n}}\left(1-\epsilon^{*}\left(\omega_{1}\right)\right)-2 C_{n}, \quad C_{n}=\int_{0}^{\infty} \frac{d \omega}{\omega} V_{n}^{2}(\omega),
$$

так что

$$
\int_{-\infty}^{+\infty} d \omega \omega V_{n}^{2}(\omega) \mathrm{P} \frac{1}{\omega_{1}-\omega}=2 \frac{\omega_{1}^{3}}{k_{1 n}}\left(1-\epsilon_{\mathrm{R}}\left(\omega_{1}\right)\right)-2 C_{n} \omega_{1} .
$$

Собирая наши результаты, мы получаем формулы

$$
\begin{aligned}
& J^{(1)}\left(\omega_{1}\right)=\frac{1}{2} \omega_{1}^{2}\left(\frac{1}{\omega_{1}}-\frac{\partial}{\partial \omega_{1}}\right) 2 \frac{\omega_{1}}{k_{1 n}}\left(1-\epsilon_{\mathrm{R}}\left(\omega_{1}\right)\right), \\
& J^{(2)}\left(\omega_{1}\right)=\frac{1}{2}\left(\frac{1}{\omega_{1}}-\frac{\partial}{\partial \omega_{1}}\right)\left[2 \frac{\omega_{1}^{3}}{k_{1 n}}\left(1-\epsilon_{\mathrm{R}}\left(\omega_{1}\right)\right)-2 C_{n} \omega_{1}\right] .
\end{aligned}
$$


Их сумма есть

$$
J\left(\omega_{1}\right)=2 \frac{\omega_{1}^{2}}{k_{1 n}}\left(\omega_{1} \epsilon_{\mathrm{R}}^{\prime}\left(\omega_{1}\right)+\epsilon_{\mathrm{R}}-1\right)=2 \frac{\omega_{1}^{2}}{k_{1 n}}\left(\left[\omega_{1}\left(\epsilon_{\mathrm{R}}\left(\omega_{1}\right)-1\right)\right]^{\prime},\right.
$$

что приводит к окончательному ответу для конечной части $F_{2}$ :

$$
F_{2}=F_{2}^{(1)}+F_{2}^{(2)}=\frac{1}{2 \pi} \int_{0}^{\infty} d \omega \omega^{2}\left[\omega\left(\epsilon_{\mathrm{R}}(\omega)-1\right)\right]^{\prime} \operatorname{Im} P_{n}(\omega) .
$$

Отметим, что последний член в $F_{2}$ сокращает первое слагаемое в $F_{0}$.

Сумма $F_{1}$ и $F_{2}$ дает равенство

$$
\begin{aligned}
\left\langle H_{Y}\right\rangle_{n} & =\frac{1}{2 \pi} \int_{0}^{\infty} d \omega \omega^{2}\left\{[\omega \operatorname{Im}(\epsilon(\omega)-1)]^{\prime} \operatorname{Re} P_{n}(\omega)+\left([\omega \operatorname{Re}(\epsilon(\omega)-1)]^{\prime}\right) \operatorname{Im} P_{n}(\omega)\right\}= \\
& =\frac{1}{2 \pi} \int_{0}^{\infty} d \omega \omega^{2} \operatorname{Im}\left([\omega(\epsilon(\omega)-1)]^{\prime} P_{n}(\omega)\right)
\end{aligned}
$$

В сумме с $\left\langle H_{A}\right\rangle_{n}$ это дает полную энергию основного состояния (35) в расчете на одну моду.

\section{Список литературы}

[1] B. Huttner, S. M. Barnett, "Quantization of the electromagnetic field in dielectrics", Phys. Rev. A, 46:7 (1992), 4306-4322.

[2] W.L. Mochán, C. Villarreal, "Casimir effect for arbitrary materials: contributions within and beyond the light cone", New J. Phys., 8 (2006), 242, 21 pp.

[3] F. C. Lombardo, F. D. Mazzitelli, A.E. Rubio López, "Casimir force for absorbing media in an open quantum system framework: scalar model", Phys. Rev. A, 84:5 (2012), 052517, $12 \mathrm{pp}$.

[4] F. S.S. Rosa, D. A.R. Dalvit, P.W. Milonni, "Electromagnetic energy, absorption, and Casimir forces: Uniform dielectric media in thermal equilibrium", Phys. Rev. A, 81:3 (2010), 033812, 12 pp., arXiv: 0911.2736.

[5] F. Interavia, R. Behunin, "Casimir effect as a sum over modes in dissipative systems", Phys. Rev. A, 86:6 (2012), 062517, 18 pp.

[6] F.S. S. Rosa, D. A. R. Dalvit, P. W. Milonni, Quantum fields in a dielectric: Langevin and exact diagonalization approaches, arXiv: 0912.0279.

[7] T. G. Philbin, "Casimir effect from macroscopic quantum electrodynamics", New J. Phys., 13 (2011), 063026, $21 \mathrm{pp}$.

[8] F.S.S. Rosa, D. A.R. Dalvit, P.W. Milonni, "Electromagnetic energy, absorption, and Casimir forces. II. Inhomogeneous dielectric media", Phys. Rev. A, 84:5 (2011), 053813, 13 pp., arXiv: 1107.4369.

[9] М. А. Браун, “Энергия Казимира квантованного поля в среде с дисперсией и поглощением", ТМФ, 175:3 (2013), 388-397.

[10] А.П. Прудников, Ю.А. Брычков, О.И. Маричев, Интеграль и ряды. Специальные функиии, Наука, М., 1983. 